\title{
Weakened Field Equations in General Relativity Admitting an 'Unphysical' Metric
}

\author{
D. LOVELOCK* \\ Department of Mathematics, University of South Africa, Pretoria \\ Received January 11, 1967
}

\begin{abstract}
Certain weakened vacuum field equations which have been suggested as alternatives to the vacuum field equations of General Relativity are investigated. It is shown that they each possess a solution which corresponds to an isolated mass at the origin which repels test particles. In view of the contradiction with experiment this solution is an embarrassment to these proposed vacuum field equations.
\end{abstract}

\section{$\S 1$. Introduction}

Our considerations are based on a four-dimensional Riemannian space with the line element ${ }^{1}$

where we assume that

$$
d s^{2}=g_{i j} d x^{i} d x^{j}
$$

$$
g \equiv \operatorname{det}\left|g_{i j}\right|<0 \text {. }
$$

In order to fix our notation we shall briefly recall certain well known definitions and results [1]. The Christoffel symbols of the second kind are defined by

$$
\left\{\begin{array}{c}
i \\
j k
\end{array}\right\}=\frac{\mathbf{1}}{2} g^{i \hbar}\left(\frac{\partial g_{h j}}{\partial x^{k}}+\frac{\partial g_{k h}}{\partial x^{j}}-\frac{\partial g_{j k}}{\partial x^{h}}\right),
$$

where the quantities $g^{i j}$ are the elements of the inverse matrix of $g_{i j}$. The curvature tensor $R_{i k l}{ }^{j}$ is introduced by means of the commutation relations

$$
X_{; k l}^{j}-X_{; l k}^{j}=R_{i k l}^{j} X^{i},
$$

where a semi-colon denotes partial covariant differentiation and $X^{i}$ is any contravariant vector field. According to this

$$
R_{i k l}{ }^{j}=\frac{\partial}{\partial x^{l}}\left\{\begin{array}{c}
j \\
i k
\end{array}\right\}-\frac{\partial}{\partial x^{k}}\left\{\begin{array}{l}
j \\
i l
\end{array}\right\}+\left\{\begin{array}{c}
h \\
i k
\end{array}\right\}\left\{\begin{array}{c}
j \\
h l
\end{array}\right\}-\left\{\begin{array}{l}
h \\
i l l
\end{array}\right\}\left\{\begin{array}{c}
j \\
k h
\end{array}\right\},
$$

and we shall define

$$
R_{i \hbar k l}=g_{j h} R_{i k l}{ }^{j} .
$$

The Ricci tensor $R_{i j}$ and the curvature scalar $R$ are defined by

$$
R_{i j}=R_{i{ }^{h} h},
$$

* On leave of absence from the Department of Mathematics, The University, Bristol.

1 The summation convention is used throughout this paper.

15 Commun. math. Phys., Vol. 5 
and

$$
R=g^{i j} R_{i j},
$$

respectively. Furthermore $R_{i k l}{ }^{j}$ satisfies the Bianchi identities

from which

$$
R_{i k l ; h}{ }^{j}+R_{i h k ; l}{ }^{j}+R_{i l h ; k}{ }^{j}=0 \text {, }
$$

where

$$
R_{j ; i}^{i}=\frac{1}{2} R_{; j}
$$

$$
R^{i}{ }_{j}=g^{i h} R_{h j} \text {. }
$$

In the orthodox theory of General Relativity the field equations are those proposed by Ensstern, viz.

$$
G_{i j}=-k T_{i j}
$$

where $G_{i j}$ is the Einstein tensor

$$
G_{i j}=R_{i j}-\frac{1}{2} g_{i j} R,
$$

$k$ is a constant and $T_{i j}$ is the energy-momentum tensor corresponding to the presence of matter in the physical situation under consideration. $G_{i j}$ enjoys the properties

and

$$
G_{i j}=G_{j i}
$$

$$
G_{j ; i}^{i} \equiv\left(g^{h i} G_{h j}\right)_{; i}=0,
$$

and the left hand side of (1.3) may be derived from a variational principle with a suitably defined Lagrange density. In vacuo

and (1.3) reduces to

$$
T_{i j}=0 \text {, }
$$

$$
R_{i j}=0
$$

These are taken as the Einstein equations in vacuum, and it is a solution of (1.4) together with the so-called geodesic hypothesis which gives agreement with experiment.

However, certain authors have proposed that the assumed vacuum field equations (1.4) be weakened by replacing them with various alternative vacuum field equations which admit (1.4) as a sub-class of solutions. In this note we consider five such suggestions and we show that they all admit the special line element

$$
d s^{2}=\frac{a^{2}}{r^{2}}\left(c^{2} d t^{2}-d r^{2}-r^{2} d \theta^{2}-r^{2} \sin ^{2} \theta d \varphi^{2}\right),
$$

where $a$ is a constant, as a solution. However, by assuming the geodesic hypothesis, we show that this metric is unphysical in the sense that it corresponds to the static situation of an isolated mass at the origin which repels test particles. Thus the alternative vacuum field equations are too weak and certain additional restrictions will have to be imposed on each of them before they become acceptable from a physical viewpoint. 


\section{$\S 2$. The Metric and its Curvature Properties}

In this section we shall derive those curvature properties of the special line element

$$
d s^{2}=\frac{a^{2}}{r^{2}}\left(c^{2} d t^{2}-d r^{2}-r^{2} d \theta^{2}-r^{2} \sin ^{2} \theta d \varphi^{2}\right),
$$

where $a$ is a constant, which we shall require in the later sections.

If we put

$$
x^{0}=c t, \quad x^{1}=r, \quad x^{2}=\theta, \quad x^{3}=\varphi,
$$

then the only non-vanishing Christoffel symbols of the second kind are

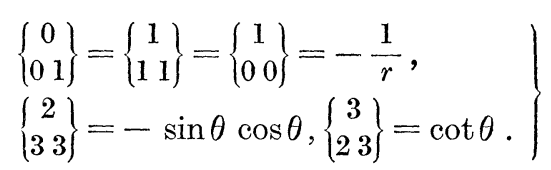

It is not difficult to calculate the components of the Ricci tensor from (2.2) and we find that

$$
\left.\begin{array}{l}
R_{00}=\frac{1}{r^{2}}, R_{11}=-\frac{1}{r^{2}}, \\
R_{22}=1, R_{33}=\sin ^{2} \theta, \\
R_{i j}=0 \text { for } i \neq j,
\end{array}\right\}
$$

while, in this case, the scalar curvature $R$ is zero, i.e.

$$
R=0 \text {. }
$$

By its very structure the metric (2.1) is conformally flat which implies that the Weyl conform tensor $C_{j \text { lim }}$, defined by

$$
\begin{aligned}
C_{j l i m}=R_{j l i m} & -\frac{1}{2}\left(R_{j i} g_{l m}-R_{l i} g_{j m}-R_{j m} g_{l i}+R_{l m} g_{i j}\right)+ \\
& +\frac{R}{6}\left(g_{i j} g_{l m}-g_{l i} g_{j m}\right),
\end{aligned}
$$

must vanish ([2], p. 306). By taking into account (2.4), (2.5) thus reduces to

$$
R_{j l i m}=\frac{1}{2}\left(R_{j i} g_{l m}-R_{l i} g_{j m}-R_{j m} g_{l i}+R_{l m} g_{j i}\right),
$$

from which it follows that

$$
R_{i h k ; j}{ }^{j}=\frac{1}{2}\left(R_{i h ; k}-R_{i k ; h}\right),
$$

by virtue of (2.4) and (1.2). However, from the Bianchi identities (1.1) we find

$$
R_{i{ }_{h k ; j}}=R_{i \hbar ; k}-R_{i k ; h} .
$$


A comparison of (2.7) and (2.8) therefore yields

or, equivalently,

$$
\left.\begin{array}{rl}
R_{i h k ; j}{ } & =0, \\
R_{i h ; k} & =R_{i k ; h}
\end{array}\right\}
$$

In fact the second equation in (2.9) may be strengthened considerably since for the metric (2.1) it can be shown that

$$
R_{i h ; k}=0 .
$$

Finally it is not difficult to show that

and

$$
\left.\begin{array}{l}
R^{i j} R_{i j}=4 / a^{2}, \\
R^{i j} R_{j}^{l}=\frac{1}{4} g^{i l}\left(R_{h_{k}} R^{h k}\right) .
\end{array}\right\}
$$

Properties (2.3), (2.4), (2.6), (2.9), (2.10) and (2.11) will be required in the next section.

\section{§ 3. Weakened Field Equations}

We now turn to certain vacuum field equations which have been suggested as alternatives to the vacuum field equations of the Einstein theory of General Relativity. We shall consider five separate cases in all, characterised by each of the following field equations:

$$
\begin{gathered}
J_{i k l} \equiv R_{i k l ; j}{ }^{j}=0, \\
\mathscr{G}_{j k} \equiv(-g)^{1 / 4}\left[g^{i n} R_{k j ; i h}-g^{i n} R_{i j ; k h}+\frac{1}{6} R_{; k j}+\right. \\
\left.-\frac{1}{6} g_{j k} g^{i n} R_{; i h}-R^{i n} C_{j h i k}+\frac{R}{6} g^{i j} C_{j h i k}\right]=0, \\
E^{h k} \equiv(-g)^{1 / 2}\left[g^{h j} g^{k i}\left\{2 R_{j l i m} R^{m l}+g^{m l} R_{i j ; l m}-R_{; i j}\right\}+\right. \\
\left.-\frac{1}{2} g^{h k}\left\{R_{m}{ }_{m} R^{m}{ }_{l}-g^{l m} R_{; l m}\right\}\right]=0, \\
\mathscr{E}^{r s} \equiv(-g)^{1 / 2}\left[\left(g^{r s} g^{t u}-\frac{1}{2} g^{r t} g^{s u}-\frac{1}{2} g^{r u} g^{s t}\right) R_{; u t}+\right. \\
\left.+R\left(R^{s r}-\frac{1}{4} g^{s r} R\right)\right]=0,
\end{gathered}
$$

and

$$
H^{i j}{ }_{k} \equiv R^{i j}{ }_{; k}=0 .
$$

All of $\mathrm{I}$ to $\mathrm{V}$ are weakened field equations (i.e. weaker than the Einstein equations in vacuo) in the sense that they each admit a class of solutions for which

$$
R_{i j}=0 .
$$


Consequently I to $\mathrm{V}$ admit the Schwarzschild metric as a solution, which when taken together with the geodesic hypothesis, gives agreement with the usual experimental tests for general relativity.

We shall not give the motivations which led authors to suggest $I$ to $V$ but merely refer to the literature cited below. Equation I was first suggested by KMLMISTER and NEwMAN [3] and has been the subject of a detailed investigation by Thомтsоn [4] who has shown that I possesses certain unphysical metrics as solutions. Equation II was proposed by Pirani [5]. The equation itself appears in Schouten ([2], p. 313, equation 6.8) in a completely different context. $\mathscr{G}_{j k}$ enjoys certain properties which one has come to expect of suitable field equations, viz.

$$
\begin{aligned}
\mathscr{G}_{j k} & =\mathscr{G}_{k j}, \\
\mathscr{G}_{k ; j}^{j} & =0,
\end{aligned}
$$

and II can be derived from a variational principle [6].

Equation III occurs in Rund [7], [8], and DU Puessis [9] and is derived from a variational principle. Although neither of these authors suggested III as a weakened field equation in General Relativity, the question was posed as to whether or not there exist solutions of III which are not solutions of

$$
R_{i j}=\mu g_{i j}
$$

VII

where $\mu$ is a constant. We also have

and

$$
E^{h k}=E^{k h},
$$

$$
E^{h k} ; \hbar=0 \text {. }
$$

Equation IV was first proposed by EDDINGTon, but has since been the subject of investigations by Buchdahl [10], Du Plessis [9] and Pechlaner and Sext [11] amongst others. It is also derivable from a variational principle and

together with

$$
\mathscr{E}^{r s}=\mathscr{E}^{s r}
$$

$$
\mathscr{E}^{\circ s} ; r=0 \text {. }
$$

Finally, using a completely different type of variational principle, RuND [12] arrived at the third order differential equations $V$. RuND has not suggested that $\mathrm{V}$ be interpreted as a weakened field equation, but, for the sake of completeness, we shall discuss it at the same time. $V$ is actually a stronger form of $\mathrm{I}$. It has also been investigated by TAKENo (see [13], p. 79) who asserts that the only spherically symmetric solution of $V$ is also a solution of

$$
R_{i j}=\mu g_{i j}
$$


We shall state the main result of this section in the form of a

Theorem. The metric

$$
d s^{2}=\frac{a^{2}}{r^{2}}\left(c^{2} d t^{2}-d r^{2}-r^{2} d \theta^{2}-r^{2} \sin ^{2} \theta d \varphi^{2}\right),
$$

where $a$ is a constant, is a solution of each of I, II, III, IV and V, but it is not a solution of VI or

where $\mu$ is a constant.

$$
R_{i j}=\mu g_{i j}
$$

We shall prove this theorem by considering $I$ to $V$ in turn, and using the results of the previous section. I follows immediately from (2.9).

$\mathrm{II}$ is a consequence of $(2.4),(2.10)$ and

$$
C_{i j \hbar l}=0 \text {. }
$$

To establish III we first calculate $R_{j l i m} R^{m l}$ from (2.4) and (2.6), obtaining

$$
R_{j l i m} R^{m l}=-R_{l i} R_{j}^{l}+\frac{1}{2} g_{i j}\left(R_{h l i} R^{h k}\right) .
$$

From the latter and (2.10) we find

$$
E^{h k}=(-g)^{1 / 2}\left[-2 R_{l} R^{l k}+\frac{1}{2} g^{h k}\left(R_{i j} R^{i j}\right)\right],
$$

which is zero by (2.11).

IV follows from (2.4) alone, while $\mathrm{V}$ is a consequence of (2.10). The proof is completed by comparing (2.3) with VI and VII.

We can therefore conclude that $I$ to $V$ are genuinely weaker than VI in the sense that spaces which are interpreted as the gravitational field in vacuo in the orthodox theory form only a proper sub-set of such spaces for the equations I to V. Furthermore, in view of the fact that the metric (2.1) is spherically symmetric, TAKENo's assertion is false.

\section{\$ 4. The "Physical" Interpretation of the Metric}

It is the purpose of this section to attempt to interpret the metric (2.1) in physical terms. Firstly we note that the usual conditions ([14], p. 104) which ensure that $t$ has the character of time and $r, \theta, \varphi$ the character of spatial coordinates are satisfied. Secondly, it is obvious that the metric (2.1) has a singularity at $r=0$. Furthermore, this singularity is not due to an unfortunate choice of coordinates since the curvature tensor also possesses this singularity in view of (2.3) and (2.6). Thus we would interpret this as an isolated mass (gravitational source) at rest at the origin for all time.

However, in order to obtain a more detailed interpretation we wish to observe the effect of this source on any test particle introduced into the system. As usual, we assume that the trajectories of test particles are 
geodesics (the so-called geodesic hypothesis), i.e. the equations of motion of such particles are given by

$$
\ddot{x}^{i}+\left\{\begin{array}{c}
i \\
j k
\end{array}\right\} \dot{x}^{j} \dot{x}^{k}=0
$$

where a dot denotes differentiation with respect to $s$. (We remark that we are not forced to accept the geodesic hypothesis in the case of the weakened field equations. However, the weakened field equations all admit the Schwarzschild metric as a solution and it is only when this is used in the geodesic equations that we have agreement with experiment. Thus, to reject the geodesic hypothesis would seem to defeat the purpose of weakening the Einstein field equations.)

By virtue of (2.2) the geodesic equations (4.1) for the metric (2.1) are

$$
\begin{aligned}
\ddot{t}-\frac{2}{r} \dot{r} \dot{t} & =0, \\
\ddot{r}-\frac{1}{r}\left(\dot{r}^{2}+c^{2} \dot{t^{2}}\right) & =0, \\
\ddot{\theta}-\sin \theta \cos \theta \dot{\varphi}^{2} & =0,
\end{aligned}
$$

and

$$
\ddot{\varphi}+2 \cot \theta \cdot \dot{\theta} \dot{\varphi}=0 .
$$

It is clear from (4.4) that if initially we have

$$
\begin{aligned}
& \theta=\pi / 2, \\
& \dot{\theta}=0,
\end{aligned}
$$

then these equations remain true for all time. But for any particular geodesic we can rotate the axes of reference so that these initial conditions hold. Thus without loss of generality we may assume

$$
\theta=\pi / 2
$$

for all time. (4.5) may then be integrated to yield

$$
\varphi=h s+b,
$$

where $h$ and $b$ are constants. Without loss of generality we may rotate the $(r, \varphi)$ plane about $\theta=\pi / 2$ so that $\varphi=0$ initially, in which case

$$
\varphi=h s \text {. }
$$

(4.2) may be integrated giving rise to

$$
\dot{t}=k r^{2}
$$

where $k$ is a constant which may be taken as positive by suitable orientation of $t$ and $s$. By substituting (4.6), (4.7) and (4.8) in (2.1) we find

$$
c^{2}-r^{\prime 2}=\frac{\alpha^{2}}{k^{2} r^{2}}
$$


where a prime denotes differentiation with respect to $t$ and

$$
\alpha^{2}=\frac{1}{a^{2}}+h^{2} .
$$

If at $t=0, r=r_{0}$ and $r^{\prime}=v$ then

$$
k=\frac{\alpha}{r_{0}\left(c^{2}-v^{2}\right)^{1 / 2}} .
$$

Finally, integration of (4.9) yields

$$
c t+\frac{r_{0} v}{c}=\left[r^{2}-r_{0}\left(1-v^{2} / c^{2}\right)\right]^{1 / 2},
$$

which shows that these equations of motion have solutions only if

$$
r^{2} \geqq r_{0}^{2}\left(1-v^{2} / c^{2}\right) .
$$

In order to obtain a more precise interpretation of these equations of motion (4.7) and (4.10), we shall restrict our considerations to those test particles which are initially (i.e. at $t=s=0$ ) released from rest at $r_{0}$. From (4.7) and (4.10) the subsequent motion will then be given by

which has solutions only if

$$
c t=\left(r^{2}-r_{0}^{2}\right)^{1 / 2}
$$

$$
r \geqq r_{0} \text {. }
$$

Hence a particle released from rest at $r_{0}$ has to move away from the origin $^{2}$ i.e. the isolated static mass at the origin repels test particles. Thus it appears that in terms of a purely gravitational interpretation the metric (2.1) is highly unphysical.

Returning to (4.11) we see that this is a hyperbola in the $(r, t)$ plane with asymptotes $r= \pm c t$. But, from (2.1), $r= \pm c t$ correspond to light rays $(d s=0)$ through the origin. Hence if a light ray is emitted from the isolated mass at the same time as the particle is released from rest, the light ray will never reach the particle in a finite time. This situation is strongly reminiscent of that of a uniformly accelerated particle in flat space-time ([15], p. 40), and in fact (4.9) (with $h=0$ ) may be rewritten in the more suggestive form

$$
\frac{d}{d t}\left[\frac{r^{\prime}}{\left(c^{2}-r^{\prime 2}\right)^{1 / 2}}\right]=\frac{1}{r_{0}\left(1-v^{2} / c^{2}\right)^{1 / 2}},
$$

which is exactly the equation of motion of a uniformly accelerated particle in flat space-time, with acceleration $1 / r_{0}\left(1-v^{2} / c^{2}\right)^{1 / 2}$.

\section{§ 5. Conclusion}

We recall the theorem proved in section 3 according to which each of the weakened vacuum field equations $I$ to $V$ admit (2.1) as a solution. However, we have also seen in section 4 that this metric cannot be

2 This is a special case of a more general theorem which is stated and proved in the Appendix. 
defended physically in the light of present experimental knowledge since it appears to correspond to the static situation of an unsupported mass which repels test particles. Hence the existence of this solution is an embarrassment to each of the proposed weakened vacuum field equations I to V. Consequently these weakened field equations are too weak and additional restrictions will have to be imposed on each of $I$ to $V$ before they become acceptable from a physical point of view.

In a subsequent paper [16] we shall discuss the interpretation of the metric (2.1) within the framework of the orthodox theory of General Relativity.

Acknowledgements. It is a pleasure to thank Professor H. Rund for the generous hospitality extended to me at the University of South Africa. I am also endebted to him for communicating the results of [12] to me before publication.

\section{Appendix}

The purpose of this Appendix is to prove the following

Theorem. If the metric of space-time is

$$
d s^{2}=\lambda\left(c^{2} d t^{2}-d r^{2}-r^{2} d \theta^{2}-r^{2} \sin ^{2} \theta d \varphi^{2}\right),
$$

where $\lambda$ is a positive, strictly monotonically decreasing function of $r$ alone, then test particles for which $d r / d s=0$ initially will always be repelled from the origin.

Proof. The geodesics (4.1) for (A 1) reduce to

$$
\begin{gathered}
\ddot{t}+\frac{\lambda_{1}}{\lambda} \dot{r} \dot{t}=0, \\
\ddot{\varphi}+\left(\frac{2}{r}+\frac{\lambda_{1}}{\lambda}\right) \dot{r} \dot{\varphi}=0,
\end{gathered}
$$

and

where

$$
1=\lambda\left(c^{2} \dot{t}^{2}-\dot{r}^{2}-r^{2} \dot{\varphi}^{2}\right)
$$

$$
\lambda_{1}=d \lambda / d r
$$

and, as usual, we have chosen our coordinates so that

$$
\theta=\pi / 2
$$

for all time. From (A2) and (A3) we find

$$
\begin{aligned}
& \dot{t}=h / \lambda, \\
& \dot{\varphi}=k / \lambda r^{2},
\end{aligned}
$$

where $h$ and $k$ are constants. Substitution of (A5) and (A6) in (A4) gives rise to

$$
\dot{r}^{2}=\left(c^{2} h^{2}-k^{2} / r^{2}-\lambda\right) / \lambda^{2} .
$$

Since initially $\dot{r}=0, r=r_{0}$ we find

$$
c^{2} h^{2}=k^{2} / r_{0}^{2}+\lambda_{0},
$$


where

$$
\lambda_{0}=\lambda\left(r_{0}\right) \text {. }
$$

However, (A7) will have solutions only if

i.e.

$$
c^{2} h^{2} \geqq k^{2} / r^{2}+\lambda
$$

$$
k^{2} / r_{0}^{2}+\lambda_{0} \geqq k^{2} / r^{2}+\lambda .
$$

Since $\lambda$ is monotonically decreasing and positive (A8) implies that $r \geqq r_{0}$ together with $\dot{r} \neq 0$ at any later time. Thus $\dot{r}>0$ and $r \geqq r_{0}$ for all time, which proves the theorem.

\section{Bibliography}

1. Synge, J. L., and A. ScHILD : Tensor calculus. Toronto: University of Toronto Press 1949.

2. Schouten, J. A.: Ricci calculus. 2nd ed. Berlin-Göttingen-Heidelberg: Springer 1954.

3. KilmisteR, C. W., and D. J. Newman: The use of algebraic structures in physics. Proc. Cambridge Phil. Soc. 57, 851-864 (1961).

4. Thompson, A. H.: The investigation of a set of weakened field equations for general relativity. Contract AF 61(052)-457 TN 10, Aerospace Research Laboratories, U.S.A.F. (1963). [This technical note is an edited form (prepared by C. W. Kilmister) of Thompson's Ph.D. thesis, University of London 1962].

5. Prrani, F. A. E.: Private communication.

6. Hesselbach, B.: Über die Erhaltungssätze der Konformen Geometrie. Math. Nachr. 3, 107-126 (1949).

7. Rund, H.: Variational problems in which the unknown functions are tensor components. Second Colloquium on the Calculus of Variations, 129-174, University of South Africa 1964.

8. - Variational problems involving combined tensor fields. Abh. Math. Sem. Univ. Hamburg 29, 243-262 (1966).

9. DU Plessis, J. C.: Invariance properties of variational principles in general relativity. Ph.D. thesis. University of South Africa 1965.

10. Buchdahl, H. A.: On the gravitational field equations arising from the square of the Gaussian curvature. Nuovo Cimento 23, 141-157 (1962).

11. Pechlaner, E., and R. Sexl: On quadratic Lagrangians in general relativity. Commun. Math. Phys. 2, 165-175 (1966).

12. Rund, H.: Invariant theory of variational problems for geometric objects. Tensor (N.S.) 19 (1967).

13. Petrow, A. S.: Einstein-Räume. Berlin: Akademie-Verlag 1964.

14. Fock, V.: The theory of space time and gravitation. London: Pergamon Press 1959.

15. Rindler, W.: Special relativity. Edinburgh: Oliver \& Boyd 1960.

16. Lovelock, D.: A spherically symmetric solution of the Maxwell-Einstein equations. Commun. math. Phys. 5, 257-261 (1967). 